\section{Post-mortem findings in vaccine-induced thrombotic thombocytopenia}

Greinacher et al. ${ }^{1}$ and Schultz et al. ${ }^{2}$ were the first to independently report the main clinical and laboratory features of 11 and five respective patients from Germany, Austria and Norway who developed life-threatening thrombohemorrhagic complications 5 to 16 days after the administration of the first dose of the chimpanzee adenoviral vector vaccine $\mathrm{ChAdOx} 1 \mathrm{nCoV}-19$ against SARS-CoV-2 and COVID-19. Subsequently Scully et al. ${ }^{3}$ reported similar findings in 23 patients treated with the same vaccine in the United Kingdom. More recently, See et al. ${ }^{4}$ reported a case series of 12 patients from the USA with cerebral venous sinus thrombosis following the vaccination with Ad26.CoV2.S employing a human adenoviral vector. The main post-vaccination features common to the case series were the occurrence of venous thromboembolism mainly in unusual sites (cerebral and abdominal veins) and the concomitant presence of bleeding symptoms associated with severe thrombocytopenia, often accompanied by laboratory signs of consumption coagulopathy with low plasma fibrinogen and hugely increased levels of $\mathrm{D}$-dimer. The majority of reported patients reacted positively for serum immunoglobulin $G$ (IgG) antibodies to the platelet factor 4 PF4/heparin complex..$^{1-4}$ Another common feature was the high mortality rate. The mechanism of this very rare thrombohemorrhagic syndrome was postulated to be a vaccine-triggered autoimmune reaction, with the development of antibodies against a still ill-defined PF4/polyanion complex that causes platelet activation as in heparin-induced thrombocytopenia (HIT) ${ }^{1-4}$ notwithstanding the fact that no cases were exposed to heparin before the onset of thrombosis and thrombocytopenia. We report herewith the detailed post-mortem macroscopic and microscopic findings in two similar cases that occurred in the Italian region of Sicily.

Case Reports. Patient 1 was a 50 -year-old man (body weight $90 \mathrm{~kg}$ ) with abdominal pain that developed 10 days after vaccination with ChAdOx1 $\mathrm{nCoV}-19$. He had neither a history for thrombosis risk factors nor had he any intake of drugs increasing this risk. At the emergency room he presented with severe thrombocytopenia, low plasma fibrinogen and very high $\mathrm{D}$-dimer (Table 1$)$. The results of other blood tests were normal except for moderately elevated white blood cells and inflammatory serum markers. Computed tomography (CT) showed portal vein thrombosis with smaller thrombi in the splenic and upper mesenteric veins. During the next 4 days after admission platelets and fibrinogen remained low and D-dimer very high with no substantial changes. An initial dose of the low molecular weight heparin nadroparin was given subcutaneously at a dosage of 5,700 IU followed by a second dose after 8 hours. Clinical conditions deteriorated and a new CT scan showed massive intracerebral hemorrhage. Treated with multiple transfusions of platelet concentrates that failed to control bleeding the patient died 4 days after the onset of symptoms and 16 days after vaccination. A serum sample obtained before nadroparin showed the presence of anti PF4/polyanion complex IgG antibodies by enzyme-linked immunosorbent assay (ELISA) (Lifecodes PF4 IgG assay, Immucor, USA).

Patient 2, a 37-year old previously healthy woman (61 $\mathrm{kg}$ ) with a negative history for significant disease and drug intake developed 10 days after the administration of the same vaccine first low back pain and then a strong
Table 1. Clinical and laboratory characteristics.

\begin{tabular}{|c|c|c|}
\hline & Case 1 & Case 2 \\
\hline Age, years & 50 & 37 \\
\hline Sex & Male & Female \\
\hline Pre-existing conditions & $\begin{array}{l}\text { None of } \\
\text { significance }\end{array}$ & $\begin{array}{l}\text { None of } \\
\text { significance }\end{array}$ \\
\hline $\begin{array}{l}\text { Time from vaccination } \\
\text { to admission, days }\end{array}$ & 10 & 10 \\
\hline Presenting symptoms & Abdominal pain & $\begin{array}{l}\text { Low back pain, } \\
\text { headache }\end{array}$ \\
\hline Thrombosis location & $\begin{array}{l}\text { Portal, splenic } \\
\text { and superior } \\
\text { mesenteric veins }\end{array}$ & $\begin{array}{c}\text { Superior sagitta } \\
\text { sinus }\end{array}$ \\
\hline Platelet count nadir, per mm $\mathrm{m}^{3}$ & 7,000 & 9,000 \\
\hline D-dimer peak, mg/dL & 52 & 290 \\
\hline Fibrinogen nadir, g/L & 0.66 & 0.34 \\
\hline Sars-CoV-2 molecular test & Negative & Negative \\
\hline Anti-PF4/polyanion complex testing & Positive for IgG & Positive for $\operatorname{IgG}$ \\
\hline Anticoagulation & Nadroparin & None \\
\hline Outcome & Fatal & Fatal \\
\hline
\end{tabular}

The reference ranges for platelet count are 130.000-400.000 per mm3, for D-dimer less than $0.5 \mathrm{mg} / \mathrm{dL}$ for fibrinogen $1.7-4.0 \mathrm{~g} / \mathrm{L}$. Ig: immunoglobulin.

headache. She became progressively drowsy and ultimately unconscious, and was, therefore, admitted to the emergency room of her local hospital. With laboratory tests similar of those of patient 1 (Table 1), a CT scan showed an occlusive thrombus in the superior sagittal venous sinus and a very large hemorrhage in the frontal cerebral lobe. Transported comatose by helicopter to a larger hub hospital she underwent craniotomy in order to control intracranial hypertension and remove the frontal lobe hemorrhage. She survived the operation but remained comatose and died 10 days after the first hospital admission and 23 days after vaccination. AntiPF4/polyanion complex antibody reactivity was detected by ELISA and confirmed in a stored serum sample (Table 1).

Autopsy findings. The anatomic dissection showed a multi-district catastrophic picture of venous thrombosis, but neither entrapment or nutcracker nor any compression which may produce blood stasis and facilitate venous thrombosis. In both cases the sites of venous thrombosis identified by imaging were confirmed, coupled with dramatic pictures of cerebral hemorrhages. While case 1 was confirmed to have had portal and mesenteric thrombosis with extension into the splenic vein, case 2 showed besides cerebral sinus thrombosis a massive thrombosis of the whole venous tree of the left upper limb extending from the hand to the axillary vein, with symmetric lesions in the veins of the right hand and the right axillary vein. In addition, the superficial veins of both feet appeared to be thrombosed. The histological evaluation revealed the presence of vascular thrombi associated with hemorrhagic phenomena localized in the meningeal space and focally involving the brain. The thrombotic phenomena also involved small- and medium-sized vessels. The immunohistochemical findings detailed in Figure 1 suggested endothelial activation associated with the dense recruitment of inflammatory myeloid cells, presumably sustaining procoagulant conditions and thrombus formation.

From a clinical and laboratory standpoint these two fatal cases of venous thrombosis located in unusual sites 
IHC
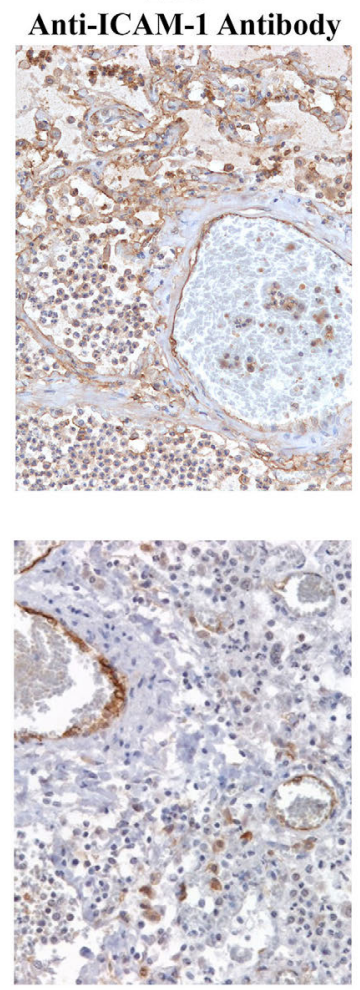

LINE 4
IHC

Anti-C4d Antibody
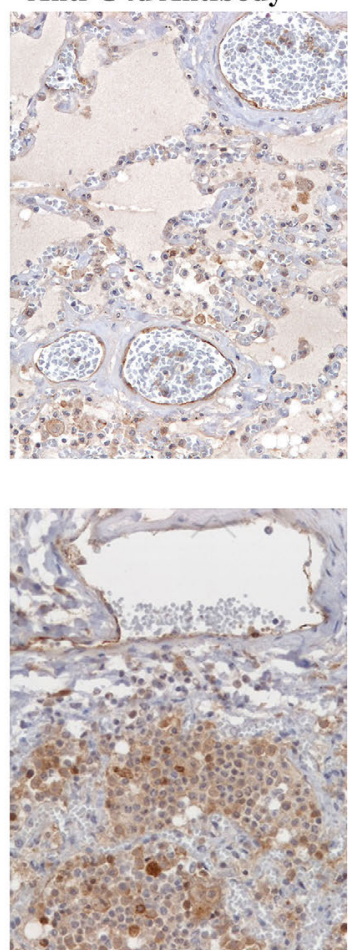

LINE 3
IHC Anti-C1r antibody
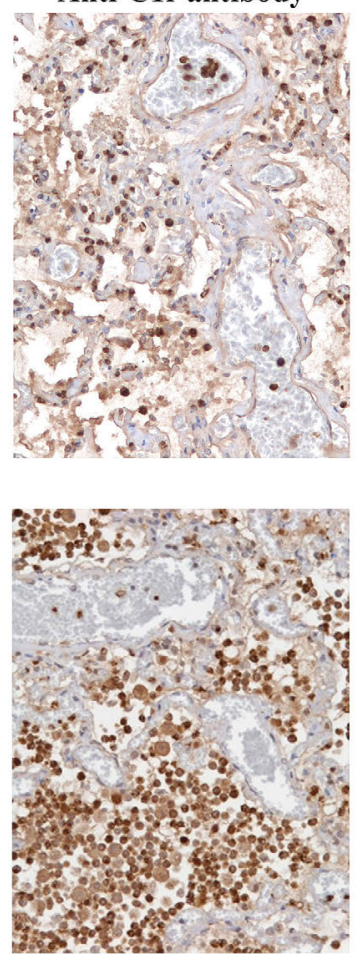

LINE 2
H\&E
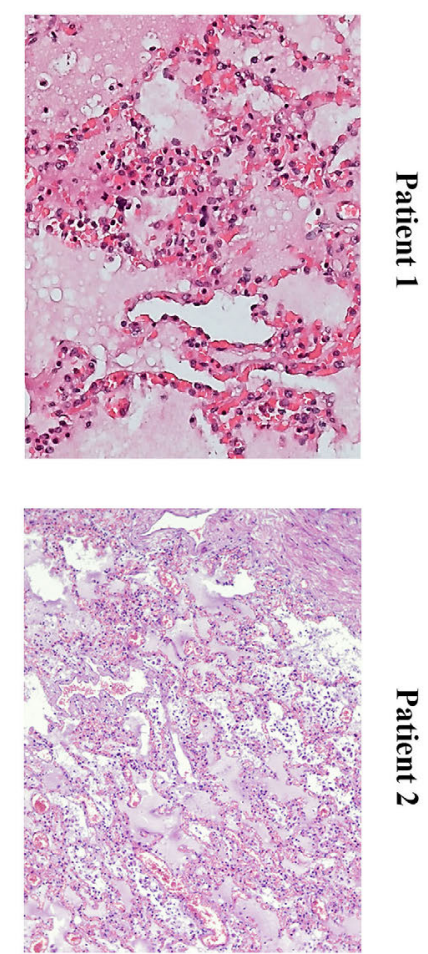

LINE 1

Figure 1. Microscopic investigation of lung tissue. (Line 1) Microscopic investigation of hematoxylin and eosin (H\&E) stained lung tissue showed marked vascular congestion, blood extravasation and the presence of microthrombi and megakaryocytes in the interstitial spaces, magnification 20X. (Line 3) Immunohistochemical staining (IHC) revealed positivity for anti-C4d antibody and anti-ICAM-1 antibody, with the presence of inflammatory cells (especially leukocytes, both polymorphonucleates and monocytes/macrophages) and the deposition of pro-inflammatory molecules (C1r, C4d) on the endothelial surface , magnification 20X.

are similar to those recently described,$^{1-4}$ because in both cases complications occurred on average 10-15 days after vaccination and were accompanied by a very low platelet count, very high D-dimer and low fibrinogen with signs of consumption coagulopathy. ${ }^{5}$ Both patients had detectable anti PF-4/polyanion antibodies unrelated to the use of heparin and positive results were confirmed by reactivity inhibition in the presence of excess heparin in vitro. ${ }^{6}$ Patients tested negative for SARS-Cov- 2 molecular assays and antibodies to the nucleocapsid and spike proteins, thus ruling out recent exposure to SARS-CoV-2 (Table 1). There was neither clinical and laboratory evidence of inherited or acquired thrombophilia nor of intake of prothrombotic medicines. Venous thrombosis was accompanied by severe intracranial bleeding, which was the final cause of death in both and developed after the administration of therapeutic doses of heparin in patient 1 but concomitantly with cerebral vein thrombosis and no anticoagulant in patient 2 .

The peculiar features of these cases were the availability of macroscopic and microscopic autopsy findings. The main macroscopic finding was that venous thrombosis was much more widespread and catastrophic than diagnosed by imaging during life. Immunohistochemistry highlighted the expression of the adhesion molecule VICAM-1 (CD106) and of the complement components $\mathrm{C} 1 \mathrm{r}$ and $\mathrm{C} 4 \mathrm{~d}$ on the vascular endothelial surface in the microcirculation of the heart, lung, liver, kidney and ileum. Diffuse endoluminal and peri-vascular immunore- activity for IgM and IgG were additional findings in the microcirculation. CD61 revealed platelet aggregates diffusely lining the endothelial layer of small- and mediumsize vessels and signs of platelet phagocytosis by myeloid elements in the vascular spaces. Scattered CD61-positive immunoreactive cells with morphological features of megakaryocytes were also detected in the lung microvasculature. The inflammation components were prominently represented by large CD163-positive monocytic/macrophagic elements that showed intra-vascular aggregates and variable monocytoid or epithelioid morphology, associated with C1r-positive medium-sized elements with granulocyte morphology.

All in all, this post-mortem examination of two typical cases of the novel vaccine-induced thrombotic thrombocytopenic syndrome (VITT) shows that the involvement of large venous vessels was much more extensive than appreciated by imaging during the brief clinical course of these fatal cases. Microscopic findings showed vascular thrombotic occlusions occurring in the microcirculation of multiple organs and increased inflammatory infiltrates. Immunohistochemical analyses highlighted the vascular and peri-vascular expression of adhesion molecules such as VICAM1, as well as the presence of $\mathrm{CD}^{2} 6 \mathrm{~b}^{+}, \mathrm{CD} 163^{+}$ and $\mathrm{CD} 1^{+}$activated inflammatory cells, also expressing C1r. These findings indicate that the activation of the innate immune system and complement pathway promote the inflammatory process leading to the microvascular damage of multiple organs. 
Cristoforo Pomara, ${ }^{1}$ Francesco Sessa, ${ }^{2}$ Marcello Ciaccio, ${ }^{3,4}$ Francesco Dieli, ${ }^{5}$ Massimiliano Esposito, ${ }^{1}$ Sebastiano Fabio Garozzo, ${ }^{6}$ Antonino Giarratano, ${ }^{7,8}$ Daniele Prati, ${ }^{9}$ Francesca Rappa, ${ }^{10}$ Monica Salerno, ${ }^{1}$ Claudio Tripodo, ${ }^{11}$ Paolo Zamboni ${ }^{12}$ and Pier Mannuccio Mannucci'

${ }^{1}$ Department of Medical, Surgical and Advanced Technologies G.F. Ingrassia, University of Catania, Catania; ${ }^{2}$ Department of Clinical and Experimental Medicine, University of Foggia, Foggia; ${ }^{3}$ nstitute of Clinical Biochemistry, Clinical Molecular Medicine and Laboratory Medicine, Department of Biomedicine, Neuroscience and Advanced Diagnostics, University of Palermo, Palermo; ${ }^{4}$ Department of Laboratory Medicine, AOUP "P. Giaccone", Palermo; ${ }^{5}$ Central Laboratory of Advanced Diagnosis and Biomedical Research (CLADIBIOR), University of Palermo, Palermo; ${ }^{6}$ Clinical Pathology Unit, Garibaldi Centro Hospital, ARNAS Garibaldi, Catania; 'Department of Surgical, Oncological and Oral Science (Di.Chir.On.S.), University of Palermo, Palermo; ${ }^{8}$ Department of Anesthesia, Intensive Care and Emergency, Policlinico Paolo Giaccone, Palermo; ${ }^{9}$ Fondazione IRCCS Ca' Granda Ospedale Maggiore Policlinico, Angelo Bianchi Bonomi Hemophilia and Thrombosis Center and Blood Transfusion Center, Milan; ${ }^{10}$ Department of Biomedicine, Neuroscience and Advanced Diagnostics, University of Palermo, Palermo; "Tumor Immunology Unit, Department of Health Sciences, University School of Medicine of Palermo, Palermo and ${ }^{12}$ Vascular Diseases Center, Mini-invasive Venous Surgery Unit, University of Ferrara, Ferrara, Italy

Correspondence: PIER MANNUCCIO MANNUCCI -

piermannuccio.mannucci@policlinico.mi.it

doi:10.3324/haematol.2021.279075
Received: April 23, 2021.

Accepted: May 13, 2021.

Pre-published: May 20, 2021.

Disclosures: no conflicts of interest to disclose.

Contributions: all authors equally contributed to the preparation of the case report.

Acknowledgments: we thank the staff of the public prosecutor office of Catania and Gela and the Department of Health of the Sicily Region for help and assistance during the post-mortem examination.

\section{References}

1. Greinacher A, Thiele T, Warkentin TE, Weisser K, Kyrle PA, Eichinger S. Thrombotic thrombocytopenia after ChAdOx1 nCov19 vaccination. N Engl J Med. 2021;384(22):2092-2101.

2. Schultz NH, Sørvoll IH, Michelsen AE, et al. Thrombosis and thrombocytopenia after ChAdOx1 $\mathrm{nCoV}-19$ vaccination. $\mathrm{N}$ Engl J Med. 2021;384(22):2124-2130.

3. Scully M, Singh D, Lown R, et al. Pathologic antibodies to platelet factor 4 after ChAdOx1 nCoV-19 vaccination. N Engl J Med. 2021;384(23):2202-2211.

4. See I, Su JR, Lale A, et al. US case reports of cerebral venous sinus thrombosis with thrombocytopenia after Ad26.COV2.S vaccination, March 2 to April 21, 2021. JAMA. 2021;325(24):2448-2456.

5. Gresele P, Marietta M, Ageno W, et al. Management of cerebral and splanchnic vein thrombosis associated with thrombocytopenia in subjects previously vaccinated with Vaxzevria (AstraZeneca): a position statement from the Italian Society for the Study of Haemostasis and Thrombosis (SISET). Blood Transfus. 2021 Apr 15 [Epub ahead of print]

6. Whitlatch NL, Kong DF, Metjian AD, Arepally GM, Ortel TL. Validation of the high-dose heparin confirmatory step for the diagnosis of heparin-induced thrombocytopenia. Blood. 2010 116(10):1761-1766 